\title{
COMPARISON COMPRESSION PROPERTIES DWT WITH FB 5/3 AND FB 9/7
}

\author{
Ondrej KOVÁČ*, Iveta GLADIŠOVÁ**, Matej ŠULA**, Volodymyr PALAHIN*** \\ *Department of Technologies in Electronics, Faculty of Electrical Engineering and Informatics, \\ Technical University of Košice, Letná 9, 04200 Košice, Slovak Republic, tel. +421 55602 3013, e-mail: ondrej.kovac@tuke.sk \\ ${ }^{* *}$ Department of Electronics and Multimedia Telecommunications, Faculty of Electrical Engineering and Informatics, \\ Technical University of Košice, Letná 9, 04200 Košice, Slovak Republic, e-mail: iveta.gladisova@tuke.sk \\ ${ }^{* * *}$ Department of Radio Engineering, Information and Telecommunication Systems, Cherkasy State Technological University, \\ Shevchenko Blvd. 460, 18006 Cherkasy, Ukraine, e-mail: palahin@yahoo.com
}

\begin{abstract}
The suitability of 2-dimensional Discrete Wavelet Transform (2D DWT) as a tool in image and video compression is nowadays indisputable. Wavelet based techniques such as JPEG2000 for image compression has a lot more to offer than conventional methods in terms of compression ratio. This paper presents DWT and the use of its compression properties in image processing and briefly describes convolutional implementation of the discrete wavelet transform. More specifically, it deals with lifting implementations of the discrete wavelet transform (LDWT). The main feature of the lifting scheme is that all constructions are derived in the spatial domain. LDWT does not require complex mathematical calculations unlike traditional methods and does not depend on Fourier transforms. The lifting scheme is used to generate second-generation wavelets, which are not necessarily translation and dilation of one particular function. This paper compares two most common types of implementation of LDWT, which are LDWT using a filter banks (FB) 5/3 and 9/7. Within the experiment, the compression properties of the LDWT implementations are compared.
\end{abstract}

Keywords: Discrete Wavelet Transform (DWT), Lifting implementation, Filter bank 5/3, Filter bank 9/7

\section{INTRODUCTION}

It is estimated that up to $80 \%$ of information is received via sight. That is why images and their processing are naturally the area of top scientific interest. The onset of integrated circuits, the development of optoelectronics, microelectronics and computing also result in an increase in digital image processing. In practice, almost all of the signals have to be processed in order to be further exploited. The digital video input data may be of different kinds. In medical technology it can be, for example, ultrasound, CT or RTG imaging. In the automotive industry, various images of parking cameras, satellite images for navigation, etc. This paper deals with a relatively modern technique of digital image processing and image analysis $[1,2]$. It is wavelet transform that is widely used in digital image processing. The paper describes one of the possible DWT uses in image processing which is compression of the image. Image compression is highly desirable nowadays, when multimedia content is on the rise and the demand for quality service is also increasing. By effective compression, it is possible to reduce the size of the data stream and thus save valuable bandwidth in the transmission channels, or to improve the quality of the data transmitted at the same bandwidth. There are several ways to reduce the amount of information in the image, and DWT is one of them [2, 3]. The following chapters briefly describe the DWT and its convolution implementation, more specifically describe the lifting implementation of the DWT (LDWT) using a filter banks (FB) 5/3 and FB 9/7 [4]. Chapter 4 compares the effectiveness of image compression LDWT with FB 5/3 and 9/7. The time consumption of both mentioned LDWT constructs is compared as well. The results of image compression effectiveness and time consumption are presented graphically at the end of the paper.

\section{DISCRETE WAVELET TRANSFORM}

The discrete wavelet transform is a transform derived from a continuous wavelet transform for discrete variables. The DWT uses a series of low-pass (LP) and high-pass (HP) filters that divide the input signal. LP filters release low frequency samples labelled "approximate", and HP filters release frequency components with higher frequencies called "detail". However, this procedure doubles the number of samples. By filtering, only the frequency spectrum is cut, not the number of samples. By omitting each second sample in both signal strings, we receive $\mathrm{N} / 2$ of detail and $\mathrm{N} / 2$ approximate samples. These samples are called detailed $(\mathrm{H})$ and approximate (L) DWT coefficients. This process is called decimation $[5,6]$. DWT is one of lossless transforms. It is recursive, i.e. the original image can be recovered using reconstruction filters inverse to the transformation filter. DWT by convolution implementation does not have integer coefficients, additional memory is needed for its realization, and it is necessary to deal with the amplification of the signal at its edges during the convolution itself. These and other disadvantages of the DWT by the convolution method remove the LDWT $[7,8]$.

\section{LIFTING IMPLEMENTATION OF DWT}

The Lifting Method is a tool for faster processing. Compared to the classical convolutional method, it is characterized by fewer memory and computational demands. It speeds up calculation and is therefore also called a fast DWT. The input discrete signal can be directly replaced by its DWT and reverse DWT can be obtained directly from the direct DWT only by changing the order of operation and signs. It allows lossless compression and has a simple technical implementation. Wavelets acquired 
using the lifting method are also called wavelets of the second generation. As mentioned above, one of the drawbacks of the classic DWT is that its coefficients are represented as floating-point numbers. This failure results in filter coefficients, which are in most cases rational or real numbers. The integrity of the LDWT coefficients is very desirable, especially for its hardware implementation. Each DWT based on lifting implementations can be easily transformed into an integer DWT (IDWT) by adding a rounding operation [9]. When speaking about IDWT, it is obvious that we mean lifting implementation, because convolutional implementation DWT cannot be an integer.

\subsection{IDWT with FB 5/3}

Filter bank (FB) $5 / 3$ belongs to the group of biorthogonal banks. FB $5 / 3$ has a relatively simple implementation, but the highest compression is achieved by filter banks with coefficients that have a large decimal fraction. It is used for lossless compression in the JPEG2000 standard. It is a filter bank that is often used in the wavelet transform by the lifting method [9]. IDWT with FB 5/3 consists of four steps: split, prediction (P), correction $(\mathrm{C})$ and rounding (symbol \llcorner\lrcorner ). Its block diagram is shown in Fig.1 [10-12].

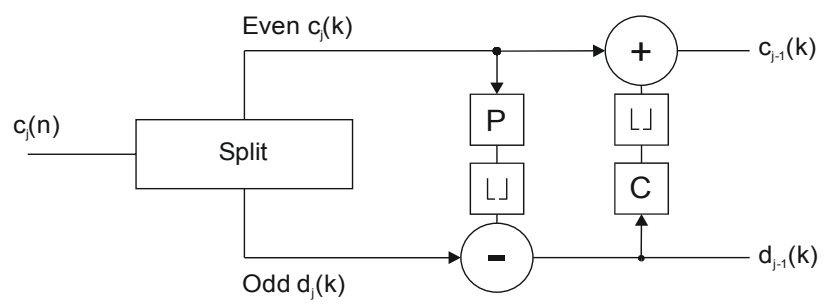

Fig. 1 Block diagram of one level decomposition using IDWT with FB 5/3

The input sequence $c_{j}(n)$ is divided into two parts. One part contains even samples $c_{j}(k)$ and the other odd samples $\mathrm{d}_{\mathrm{j}}(\mathrm{k})$. As the odd and even samples are highly correlated, the sequence of detail coefficients can be calculated as difference between data of odd part and the result of the $\mathrm{P}$ (prediction) unit. After prediction of even samples, a rounding operation is used. It is clear that if the input is an integer, after subtraction of the rounded predict samples from integer odd samples, the detail coefficients will be integers. This gives the detail coefficients $d_{j-1}(k)$. Finally, based on the detail coefficients the value of the correction is computed, it is also rounded and added to the even samples, thus we get the approximate coefficients $c_{j-1}(k)$ [10-13]. The output of one step of decomposition of 2D IDWT with FB 5/3 is described by equations (1) a (2).

$$
\begin{aligned}
& d_{j-1}(k)=d_{j}(k)-\left\lfloor 0.5\left(c_{j}(k)+c_{j}(k+1)\right)\right\rfloor \\
& c_{j-1}(k)=c_{j}(k)+\left\lfloor 0.25\left(d_{j-1}(k)+d_{j-1}(k-1)\right)\right\rfloor
\end{aligned}
$$

By applying these steps separately to the lines and image columns, a two-dimensional image decomposition using IDWT is obtained. The process of such decomposition is shown in Fig. 2 and a block diagram in Fig. 3.

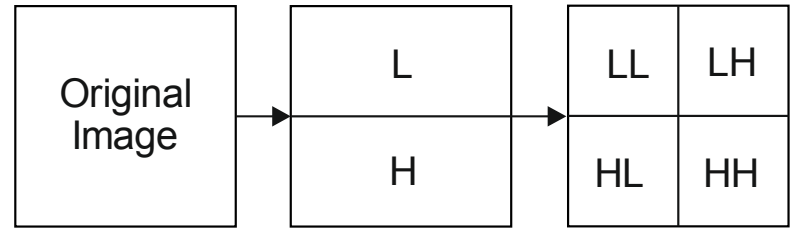

Fig. 2 The 2D IDWT decomposition achieved by application of 1D IDWT on rows and columns separately

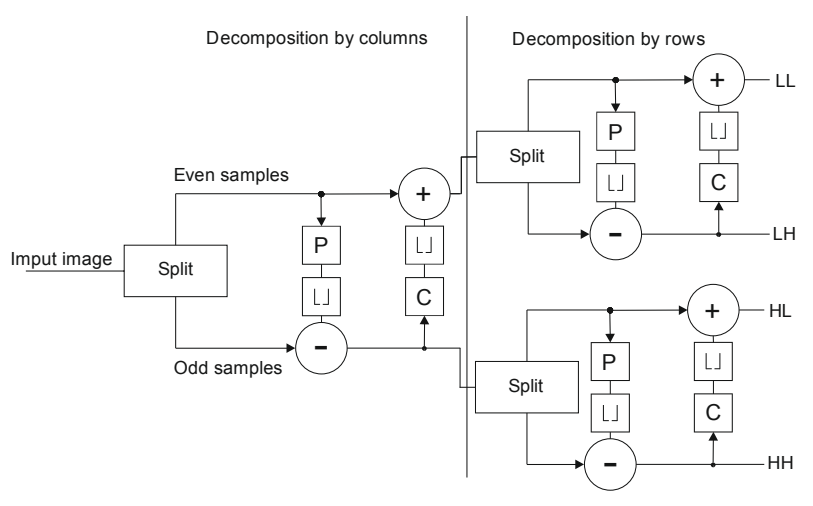

Fig. 3 Block diagram of one level decomposition using 2D IDWT with FB 5/3

\subsection{IDWT with FB 9/7}

Filter bank $9 / 7$ belongs as well as $\mathrm{FB} 5 / 3$ to a biorthogonal group and is also often used for lifting wavelet transforms. It is used in the JPEG2000 standard for lossy compression. Its implementation is more complex compared to $\mathrm{FB} 5 / 3$ and its computational demand is higher, but it achieves better results. IDWT with FB 9/7 is made up of the same steps as FB 5/3, but the predictive and correction steps are repeated twice [10-13]. The IDWT block diagram with FB 9/7 is shown in Fig. 4.

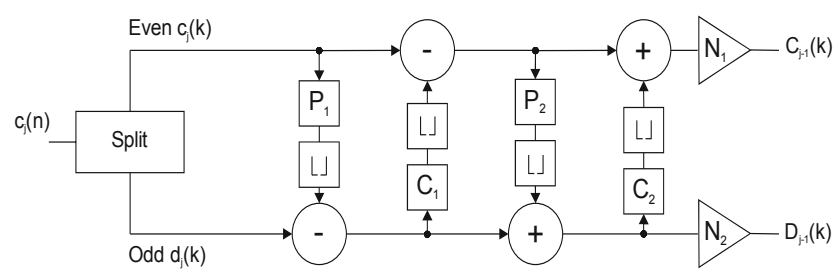

Fig. 4 Block diagram of one level decomposition using IDWT with FB 9/7

The input sequence $c_{j}(n)$ is split into two branches. One contains even samples $c_{j}(k)$ and the other odd samples $d_{j}(k)$. In the second step, the predicted value is calculated based on the even samples. It is rounded and subtracted from odd samples. The corrected value is calculated and rounded using odd samples and is added to even samples. In this way, we get the approximate $c_{j m}$ and the detail $d_{j m}$ coefficients for the intermediate level. By repeating the prediction and correction steps, the resulting approximate $\mathrm{c}_{\mathrm{j}-1}(\mathrm{k})$ and detail coefficients $\mathrm{d}_{\mathrm{j}-1}(\mathrm{k})$ [10-13] are calculated. The resulting approximation and detail coefficients have yet to be normalized using the normalizing constants $\mathrm{N}_{1}$ and $\mathrm{N}_{2}$. The resulting coefficients are denoted as $\mathrm{D}_{\mathrm{j}-1}$ and $\mathrm{C}_{\mathrm{j}-1}$. The output of one step of decomposition 2D IDWT with FB 9/7 is described by equations (3) - (8). 


$$
\begin{aligned}
& d_{j m}(k)=d_{j}(k)-\left\lfloor 1,58613\left(c_{j}(k)+c_{j}(k+1)\right)\right\rfloor \\
& c_{j m}(k)=c_{j}(k)-\left\lfloor 0,05298\left(d_{j m}(k)+d_{j m}(k-1)\right)\right\rfloor \\
& d_{j-1}(k)=d_{j m}(k)+\left\lfloor 0,88291\left(c_{j m}(k)+c_{j m}(k+1)\right)\right\rfloor \\
& c_{j-1}(k)=c_{j m}(k)+\left\lfloor 0,44351\left(d_{j-1}(k)+d_{j-1}(k-1)\right)\right\rfloor \\
& D_{j-1}(k)=1,149604 d_{j-1}(k) \\
& C_{j-1}(k)=\frac{c_{j-1}(k)}{1,149604}
\end{aligned}
$$

\section{EXPERIMENTAL RESULTS}

For the simulation of 2D IDWT with FB 5/3 and FB 9/7, an algorithm was developed in the MATLAB R2015a programming environment. Four standard test pictures were used for simulations: Lena, Baboon, Barbara and Peppers shown in Fig.5. All images were in 8-bit greyscale and resolution of $1024 \times 1024$ pixels.

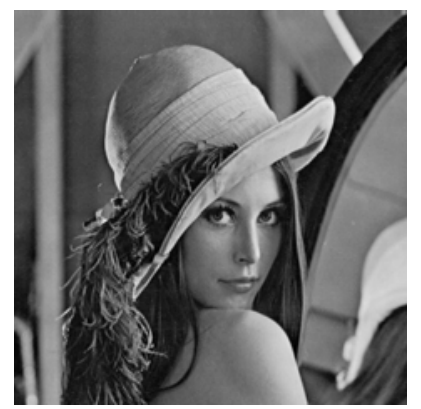

a)

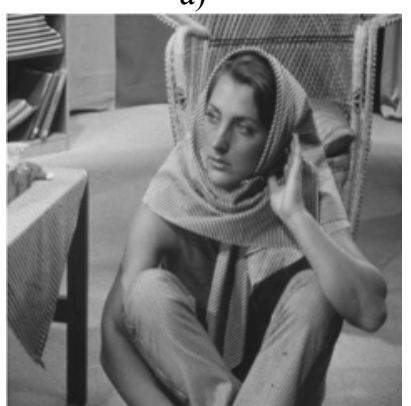

c)

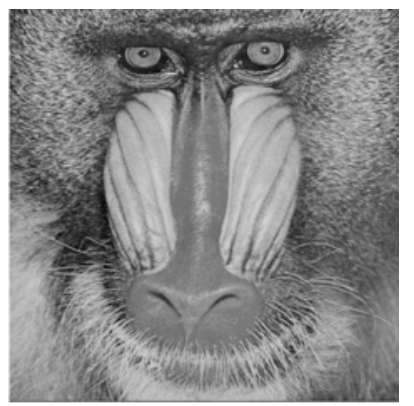

b)

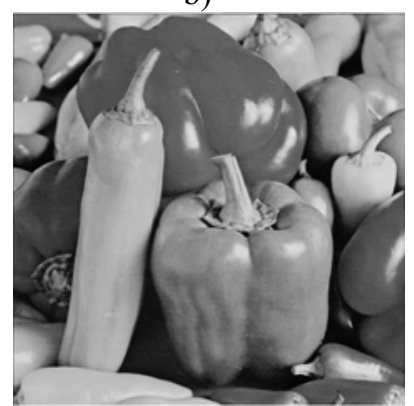

d)
Fig. 5 Pictures used in simulation.

a) Lena, b) Baboon, c) Barbara and d) Peppers

For simulation of FB 5/3 and FB 9/7 compression properties, the measured factor was the amount of energy present in the LL subband in proportion to the total image energy expressed in percent. The selected standard images were sequentially decomposed using FB 5/3 and then using FB 9/7. The compression efficiency results for FB 5/3 are shown in Fig. 6 and Table. 1 and the compression efficiency results for FB $9 / 7$ are shown in Fig. 7 and Table 2.

Table 1 Amount of energy in LL subband for IDWT with FB $5 / 3$

\begin{tabular}{ccccc}
\hline \hline $\begin{array}{c}\text { Decomposition } \\
\text { level }\end{array}$ & $\begin{array}{c}\text { Lena } \\
{[\%]}\end{array}$ & $\begin{array}{c}\text { Baboon } \\
{[\%]}\end{array}$ & $\begin{array}{c}\text { Barbara } \\
{[\%]}\end{array}$ & $\begin{array}{c}\text { Peppers } \\
{[\%]}\end{array}$ \\
\hline $\mathbf{1}$ & 95.542 & 90.285 & 91,046 & 96,933 \\
$\mathbf{2}$ & 78.391 & 64.224 & 64,306 & 84,636 \\
$\mathbf{3}$ & 44.071 & 29.803 & 29,187 & 54,410 \\
$\mathbf{4}$ & 15.347 & 9.445 & 9,022 & 21,852 \\
$\mathbf{5}$ & 4.131 & 2.516 & 2,384 & 6,329 \\
$\mathbf{6}$ & 1.065 & 0.606 & 0,575 & 1,556 \\
$\mathbf{7}$ & 0.238 & 0.1431 & 0,143 & 0,401 \\
\hline \hline
\end{tabular}

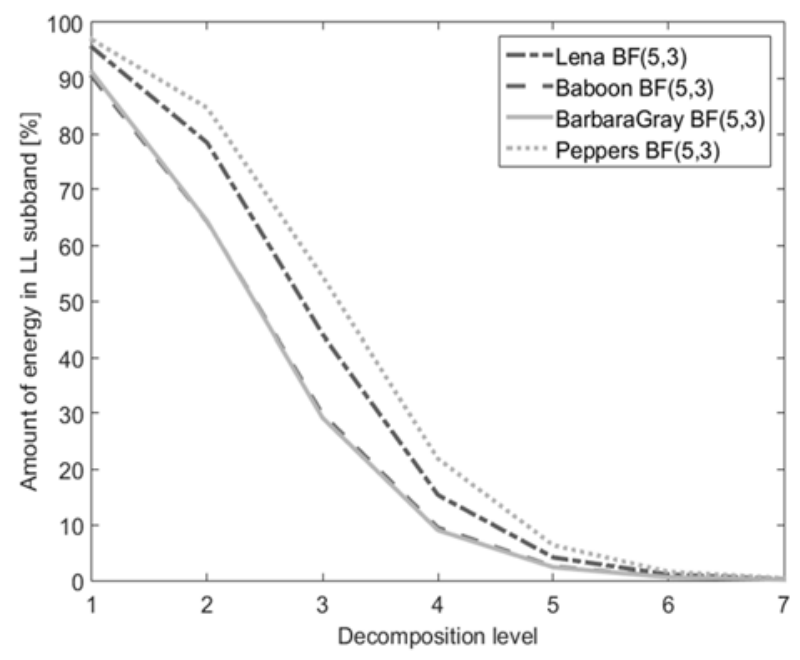

Fig. 6 Comparison of energy compression efficiency to LL subband for different pictures with FB 5/3

Table 2 Amount of energy in LL subband for IDWT with FB 9/7

\begin{tabular}{ccccc}
\hline \hline $\begin{array}{c}\text { Decomposition } \\
\text { level }\end{array}$ & $\begin{array}{c}\text { Lena } \\
{[\%]}\end{array}$ & $\begin{array}{c}\text { Baboon } \\
{[\%]}\end{array}$ & $\begin{array}{c}\text { Barbara } \\
{[\%]}\end{array}$ & $\begin{array}{c}\text { Peppers } \\
{[\%]}\end{array}$ \\
\hline $\mathbf{1}$ & 97.893 & 95.415 & 95,807 & 98,533 \\
$\mathbf{2}$ & 89.413 & 81.671 & 81,909 & 92,513 \\
$\mathbf{3}$ & 65.161 & 51.926 & 51,124 & 73,139 \\
$\mathbf{4}$ & 30.174 & 21.051 & 19,959 & 38,937 \\
$\mathbf{5}$ & 9.232 & 6.188 & 5,712 & 13,326 \\
$\mathbf{6}$ & 2.403 & 1.554 & 1,433 & 3,530 \\
$\mathbf{7}$ & 0.573 & 0.373 & 0,350 & 0,917 \\
\hline \hline
\end{tabular}

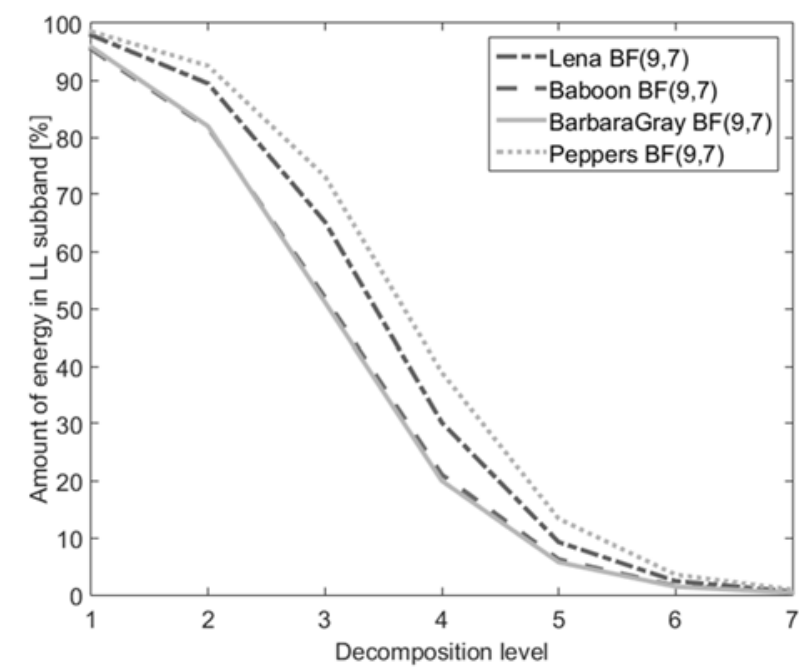

Fig. 7 Comparison of energy compression efficiency to LL subband for different pictures with FB 9/7

The results above show that the compression level is different for each image. For both lifting implementations of IDWT, the tested images Lena and Peppers showed a higher level of compression than images Barbara and Baboon. This is due to the fact that images with fewer details and edges show a higher correlation and thus greater redundancy. Transformation (FB 5/3 used) of images Lena and Barbara are shown in Fig. 8 and normalized energy distribution of these transformed images are shown in Fig. 9. From these figures can be seen that in detail subimages of image Barbara is stored more energy than in the case of image Lena. 


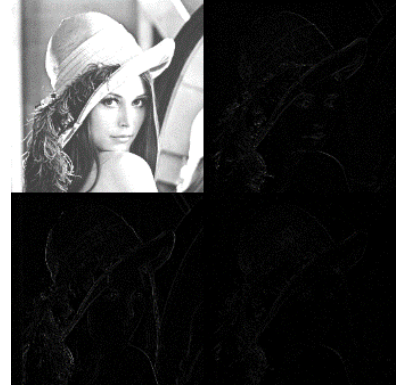

a)

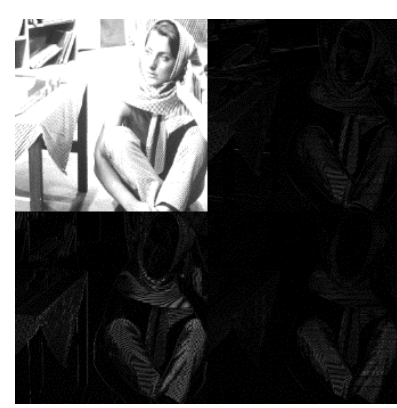

b)
Fig. 8 IDWT of images a) Lena and b) Barbara

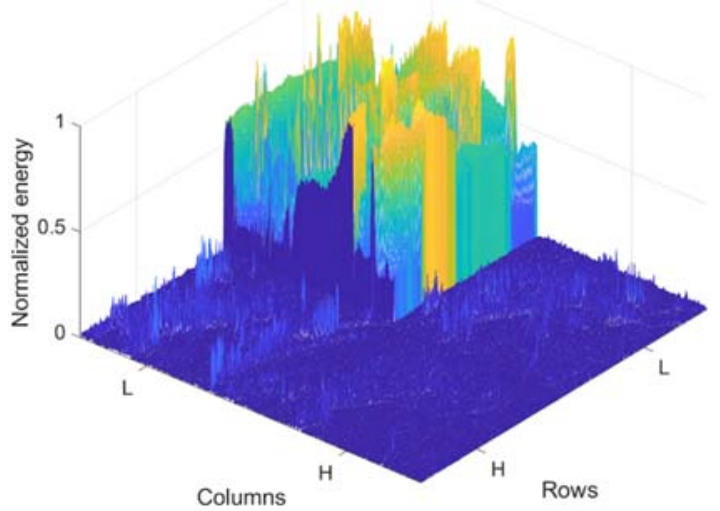

a)

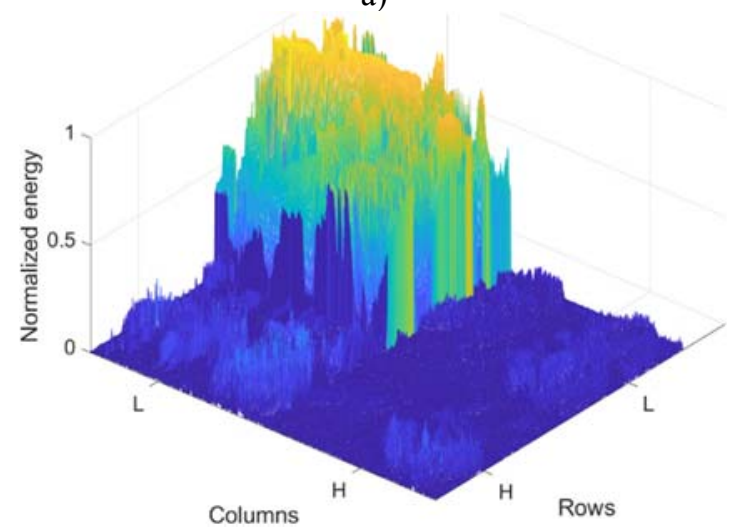

b)

Fig. 9 Normalized energy distribution for images a) Lena and b) Barbara

To compare the compression properties of both FBs tested, the average of the energy values of 40 images was calculated. These images are mainly textures [14] and images mentioned in the beginning of this chapter. Table 3 shows average values and deviations.

Table 3 Average values of energy in the LL subband

\begin{tabular}{ccccc}
\hline \hline Level & $\begin{array}{c}\text { FB(5,3) } \\
{[\mathbf{\%}]}\end{array}$ & $\begin{array}{c}\text { STD } \\
{[\%]}\end{array}$ & $\begin{array}{c}\text { FB(9,7) } \\
{[\%]}\end{array}$ & $\begin{array}{c}\text { STD } \\
{[\%]}\end{array}$ \\
\hline $\mathbf{1}$ & 89.1520 & 8.5585 & 89.5635 & 8.6468 \\
$\mathbf{2}$ & 64.1603 & 19.1225 & 70.7515 & 17.8382 \\
$\mathbf{3}$ & 33.8842 & 19.4911 & 46.3323 & 21.3147 \\
$\mathbf{4}$ & 12.9861 & 11.1896 & 24.6008 & 16.9905 \\
$\mathbf{5}$ & 3.8753 & 4.0242 & 10.7197 & 9.5518 \\
$\mathbf{6}$ & 1.0265 & 1.1503 & 4.0361 & 4.0992 \\
$\mathbf{7}$ & 0.2655 & 0.3097 & 1.4261 & 1.5579 \\
\hline \hline
\end{tabular}

The graph in Fig. 10 shows that the FB 9/7 achieves higher compression characteristics during the compression energy to LL subband compared with FB 5/3 for all tested levels of decomposition.

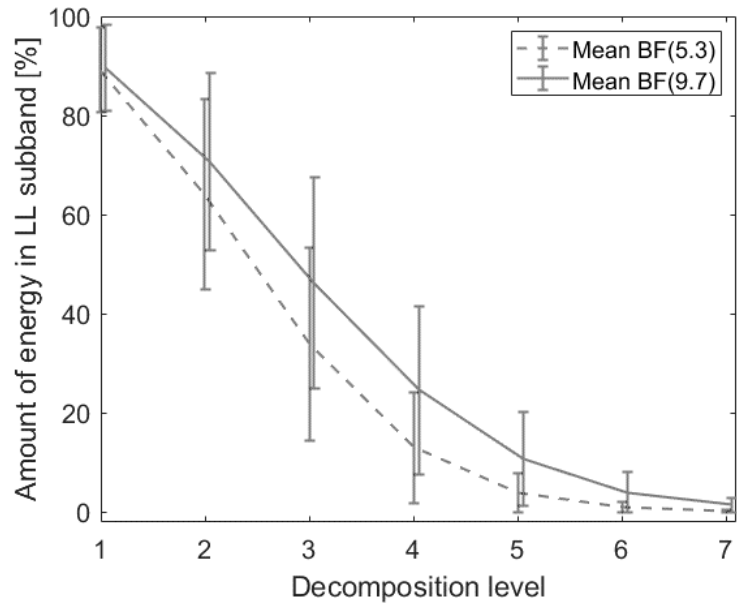

Fig. 10 Comparison of energy compression efficiency to LL band for FB 5/3 and FB 9/7

As stated in the theory above, the FB 5/3 algorithm consists of fewer steps and is therefore faster than the FB 9/7 algorithm. Simulation to compare the speed of algorithms ran 50 times for experimental images (Lena, Baboon, Barbara, Peppers) and both tested filter banks. The simulation was performed with a computer with these parameters: CPU - Intel Core i5-3210M 2.5GHz, RAM 8GB, HDD - 1TB $5400 \mathrm{rpm}$ and OS - Windows 10. Fig. 11 and Table. 4 give the average times, standard deviation and trend curves at both FB for different levels of decomposition. The graph shows that FB 9/7 at all levels of decomposition is actually slower than FB 5/3, and both curves have an increasing trend with the increasing levels of decomposition.

Table 4 Duration of algorithms

\begin{tabular}{ccccc}
\hline $\begin{array}{c}\text { Decomposition } \\
\text { level }\end{array}$ & $\begin{array}{c}\text { FB } \\
\mathbf{5 / 3}[\mathbf{s}]\end{array}$ & $\begin{array}{c}\text { Deviation } \\
{[\mathbf{s}]}\end{array}$ & $\begin{array}{c}\text { FB } \\
\mathbf{9 / 7}[\mathbf{s}]\end{array}$ & $\begin{array}{c}\text { Deviation } \\
{[\mathbf{s}]}\end{array}$ \\
\hline $\mathbf{1}$ & 0,0676 & 0,0098 & 0,0963 & 0,0128 \\
$\mathbf{2}$ & 0,0636 & 0,0052 & 0,1111 & 0,0105 \\
$\mathbf{3}$ & 0,0698 & 0,0064 & 0,1139 & 0,0047 \\
$\mathbf{4}$ & 0,0754 & 0,0086 & 0,1337 & 0,0142 \\
$\mathbf{5}$ & 0,0720 & 0,0051 & 0,1235 & 0,0091 \\
$\mathbf{6}$ & 0,0818 & 0,0152 & 0,1327 & 0,0212 \\
$\mathbf{7}$ & 0,0858 & 0,0107 & 0,1610 & 0,0180 \\
$\mathbf{8}$ & 0,0805 & 0,0069 & 0,1235 & 0,0197 \\
$\mathbf{9}$ & 0,0785 & 0,0060 & 0,1427 & 0,0225 \\
\hline \hline
\end{tabular}

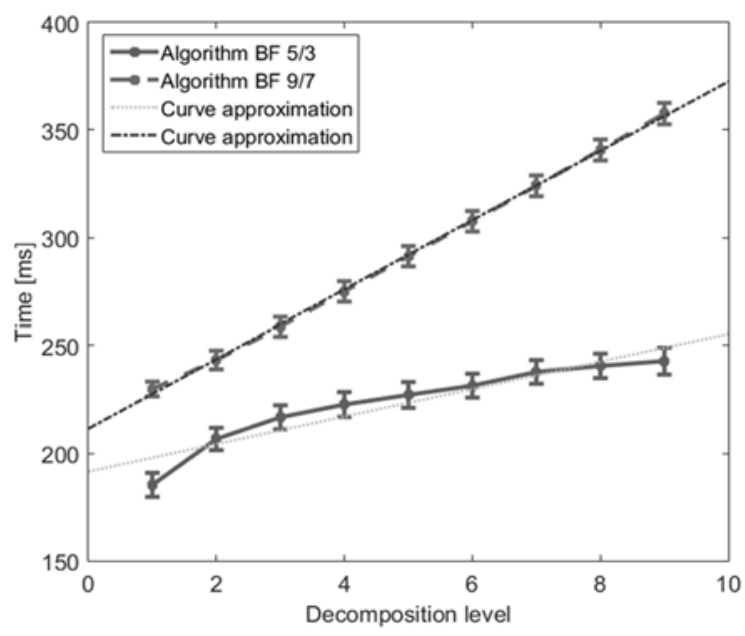

Fig. 11 Duration of algorithms 


\section{CONCLUSIONS}

The aim of this paper was to describe the structure and properties of DWT, its use in compressing images as well as to compare compression properties of the two most popular filter banks, FB 5/3 and FB 9/7. It provides a brief description of the convolution method of DWT and the detail is given to IDWT method, specifically to its integer form using the above-mentioned FB. In the simulations, four basic test images were gradually decomposed using 2D IDWT with FB 5/3 and FB 9/7: Lena, Baboon, Barbara and Peppers. The observed factor was the amount of energy in the LL subband in proportion to the total energy of the decomposed image. The results show that images with fewer details and edges show higher compression properties than more complex images. The compression properties of IDWT with FB 5/3 and 9/7 were also statistically evaluated by transformation of 40 images. It was shown that FB 9/7 achieved higher compression properties compared to FB 5/3. Finally, it was confirmed by the simulation that the more complicated FB 9/7 algorithm is actually slower than the FB $5 / 3$ algorithm for all tested decomposition levels.

\section{ACKNOWLEDGMENTS}

This paper was supported by grant no. FEI-2017-38.

\section{REFERENCES}

[1] ANTONIOU, A.: Digital signal processing. McGrawHill, 2016.

[2] JHNE, B..: Digital image processing, Springer Publishing Company, Incorporated, 2012.

[3] FLIEGE, N., L.: Multirate digital processing: Multirate systems, Filter Banks, Wavelets. West Sussex England: John Willey \& Sons, 1999.

[4] VARGIC, R.: Wavelety a banky filtrov. Bratislava. STU-FEI, 2004.

[5] YUN-NAN, CH. - YAN-SHENG, L.: Design of highly efficient VLSI architectures for 2-D DWT and 2-D IDWT. IEEE Workshop on Signal Processing Systems, 26-28, 2001.

[6] CROCHIERE, R. - RABINER, L.: Optimum FIR digital filter implementations for decimation, interpolation, and narrow-band filtering. IEEE Transactions on Acoustics, Speech, and Signal Processing, 444-456, 1975.

[7] JUNG, G., Ch. - JIN, D. Y. - PARK, S. M.: An efficient line based VLSI architecture for 2D lifting DWT, Circuits and Systems, 2004.

[8] KOVÁČ, O. - MIHALÍK, J. - GLADIŠOVÁ, I.: Convolution implementation with a novel approach of DGHM multiwavelet image transform, Journal of Electrical Engineering. Vol. 68, No. 6, s. 455462, 2017.

[9] ANTONINI, M - BARLAND, M. - MATHIEU, P. DAUBECHIES, I.: Image Coding Using Wavelet
Transform. IEEE Trans. Image Process., Vol. 1, No. 2, p. 205-220, 1992.

[10] DAUBECHIES, I. - SWELDENS, W.: Factoring Wavelet Transforms into Lifting Steps. J. Fourier Anal. Appl., Vol. 4, No. 3, p. 247-269, 1998.

[11] SWELDENS, W.: The Lifting Scheme: A Construction of Second Generation Wavelets. Siam J. Math. Anal., Vol. 29, No. 2, p. 511-546, 1997.

[12] SWELDENS, W.: The Lifting Scheme: A New Philosophy in Biorthogonal Wavelet Constructions. Proc. SPIE 2569, p. 68-79, 1995.

[13] ANDRA, K. - CHAKRABARTI, C. - ACHARYA, T.: A VLSI architecture for lifting-based forward and inverse wavelet transformll, IEEE Trans. Signal Processing, Vol. 50, No. 4, pp. 966-977, 2002.

[14] KOVAC, O. - LUKACS P. - GLADISOVA, I.: Textures classification based on DWT, 2018 28th International Conference Radioelektronika (RADIOELEKTRONIKA), Prague, pp. 1-5, 2018.

Received Aprile 19, 2018, accepted October 2, 2018

\section{BIOGRAPHIES}

Ondrej Kováč graduated from the Technical University of Košice in 2011. He received the Ing. degree in Multimedia telecommunications from the Faculty of Electrical Engineering, Technical University of Košice. His PhD. thesis topic was focused to texture generating, 3D modelling and coding of human head. Since June 2015, he has been working as assistant professor at the Department of Technologies in Electronics, Technical University of Kosice.

Iveta Gladišová graduated from the Technical University of Košice in 1984. She is employed at same university where received her $\mathrm{PhD}$ Degree in Radioelectronics in 1997. She is Assistant Professor at the Department of Electronics and Multimedia Telecommunications, Faculty of Electrical Engineering and Informatics, Technical University of Košice. Her research interests include entropy coding, image segmentation, vector quantization and digital image processing.

Matej Šul'a in 2017 received the Ing. degree in Multimedia telecommunications from the Faculty of Electrical Engineering, Technical University of Košice. His Ing. thesis topic was focused on the use of DWT in image processing.

Volodymyr Palahin graduated from Kiev polytechnic Institute (NTUU "KPI") in 1992. He received his Ph.D. degree in Radioengineering from the Odesa Nationality Polytechnical University (Ukraine) in 1999 and Dr.Sc. degree in mathematical modelling from Pukhov Institute for Modelling in Energy Engineering (Nationality Academy of Science of Ukraine) in 2014. He was appointed professor at the Department of 
RadioEngineering, Telecommunication and Robotic processing, theoretical statistics and mathematics in Systems (Cherkasy State Technological University) in practice applications - signals detection and recognition, 2015. Currently he works as an Head at this Department. parameters estimation in electrical engineering. His research interests include the integration of signal 